\title{
Idiopathic Esophageal Lipomatosis: An Incidental Benign Discovery
}

\author{
Venkata Vinod Kumar Matlia $^{a}$ Deepthi Devagudi ${ }^{b} \quad$ Brian Cooney $^{c}$ \\ Uma Murthy ${ }^{d}$ \\ aDepartment of Internal Medicine, SUNY Upstate Medical University, Syracuse, NY, USA; \\ ${ }^{\text {b}}$ Department of Internal Medicine, West Anaheim Medical Center, Anaheim, CA, USA; \\ 'Department of Radiology, Syracuse Veteran Affairs Medical Center, Syracuse, NY, USA; \\ dDepartment of Gastroenterology, Syracuse Veteran Affairs Medical Center, Syracuse, NY, \\ USA
}

\section{Keywords}

Esophageal adiposis · Computed tomography · Incidental findings · Esophageal lipoma ·

Gastrointestinal lipoma

\begin{abstract}
Gastrointestinal (GI) lipomatosis has been reported in the GI medicine literature, but esophageal lipomatosis has never been reported at all. We report the case of an 86-year-old man with multiple medical comorbidities who was admitted to our hospital for community-acquired pneumonia. Computed tomography angiography of his pulmonary arteries ruled out the possibility of pulmonary embolism but showed a 9-mm circumferential wall thickening in the proximal esophagus measuring $-172 \mathrm{HU}$, which is similar in opacity to the adipose tissue. The patient was asymptomatic and without any current or prior symptoms of dysphagia or odynophagia. The barium esophagogram was unremarkable; there were no strictures, masses, or mucosal abnormalities. There was no evidence of esophageal dilatation on either imaging modality. Esophageal lipomatosis is only described in a few case reports in the radiological literature and, to our knowledge, has not been reported in the Gl literature at all. It is important to highlight in the $\mathrm{Gl}$ literature this as a benign entity that does not cause symptoms and typically does not warrant invasive diagnostic or therapeutic interventions.
\end{abstract}

\section{Karger!}




\section{Introduction}

The term adiposis or lipomatosis refers to the proliferation of mature adipocytes in soft tissues. Diffuse gastrointestinal (GI) lipomatosis in the small and large intestines is a wellrecognized entity associated with chronic inflammatory bowel disease particularly Crohn's disease. It may also occur in patients with graft-versus-host disease, celiac disease, and radiation enteritis. Lipomatosis of other organs including the pancreas, gallbladder, liver, kidneys, and heart has also been reported in the literature. Mediastinal lipomatosis has been reported in patients undergoing chronic steroid therapy, in those with Cushing's syndrome, and in those with extreme obesity. In the radiological literature, however, esophageal lipomatosis has only been described in a few case reports and, to our knowledge, has not been reported in the GI literature at all. Here, we describe an incidental finding of esophageal lipomatosis using computed tomography (CT) imaging. It is important to highlight that the findings report esophageal lipomatosis as a benign entity that does not cause adverse symptoms and usually does not warrant invasive diagnostic or therapeutic interventions.

\section{Case Report/Case Presentation}

An 86-year-old man with multiple comorbidities, including diabetes mellitus, chronic obstructive pulmonary disease (COPD), coronary artery disease, hypertension, severe aortic stenosis with prior valve replacement, congestive heart failure, and obesity (BMI of 35), was hospitalized with community-acquired pneumonia. A thoracic CT scan with contrast was performed to evaluate the possibility of pulmonary embolism (shown in Fig. 1). This revealed an incidental finding of a 9-mm circumferential wall thickening in the proximal esophagus measuring $-172 \mathrm{HU}$. The low attenuation rings were smooth, well demarcated, and similar in opacity to adipose tissue. The higher attenuation ring in the middle was similar in density to the adjacent muscle tissue. The patient did not have any current or prior symptoms of dysphagia or odynophagia. The barium esophagogram was unremarkable; there were no strictures, masses, or mucosal abnormalities (shown in Fig. 2). There was no evidence of esophageal dilation in either imaging modality. The patient had no previous upper endoscopies, nor had he been previously treated with corticosteroids. The decision to clinically observe and monitor the patient for any developing symptoms was made, and no further evaluation of the esophagus was pursued.

\section{Discussion}

Intestinal lipomatosis is very rare, with a reported incidence at autopsy ranging from $0.04 \%$ to $4.5 \%$ [1], and is associated with inflammatory bowel diseases, particularly Crohn's disease. This deposition or infiltration of mature adipose tissue in the submucosal layer of the intestine is thought to be because of an underlying chronic inflammatory process. Diffuse GI lipomatosis involving the stomach and small and large intestines has been described in the GI literature [2]. The most common sites for GI lipomatosis are the cecum and ileum. The potential complications of intestinal lipomatosis are GI bleeding, as described in a case study of an erosive duodenal lipoma [3], intussusception [4], obstruction, and perforation leading to peritonitis [5].

Benign esophageal tumors, such as esophageal lipoma, are typically asymptomatic and rarely occur, with an incidence of $<1 \%$ [6]. They grow very slowly and can remain stable in size for prolonged periods of time. As such, symptoms of dysphagia often occur late in disease

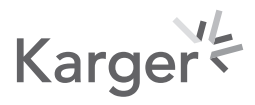


Fig. 1. CT of the thorax with intravenous contrast showing concentric rings of enhancement due to fatty infiltration of the esophagus ("target esophagus"). CT, computed tomography.

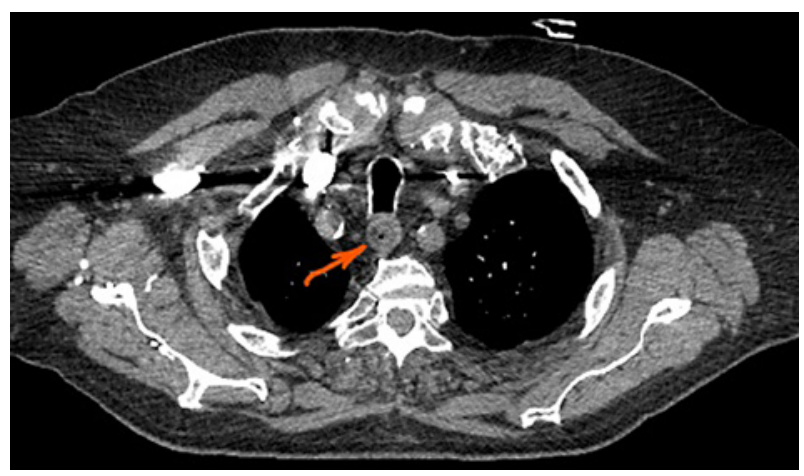

Fig. 2. Barium esophagogram: no strictures observed, and flexibility of the esophagus was intact.

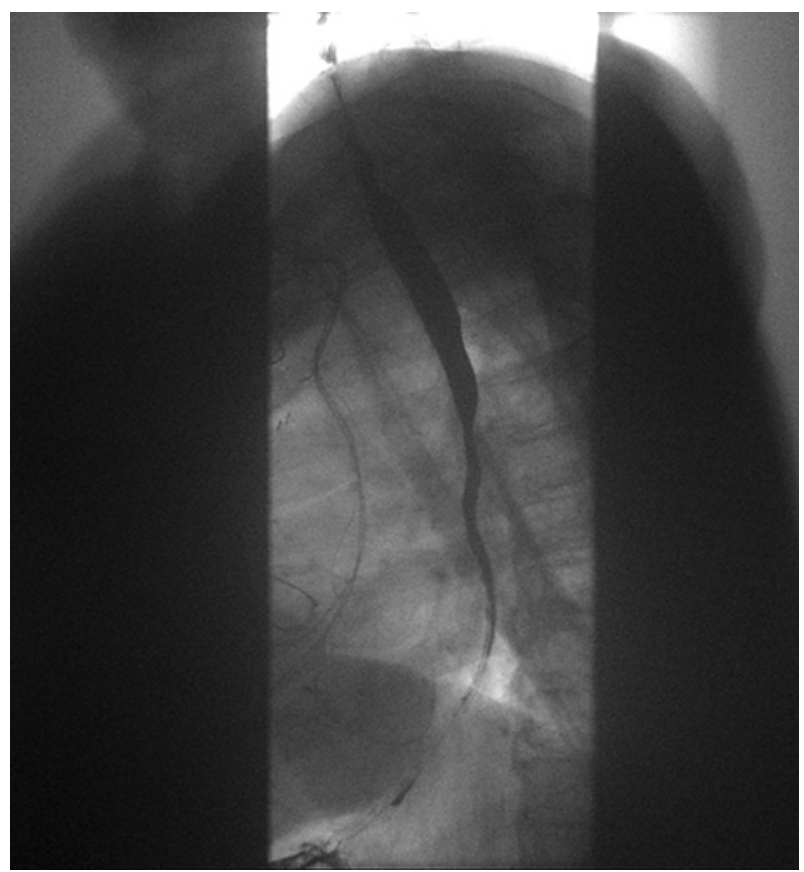

progression as lesions grow large enough (usually $>5 \mathrm{~cm}$ ) to cause endoluminal compression or obstruction. Most commonly, benign esophageal tumors are found incidentally on imaging. If the tumor is symptomatic, endoscopy or endoscopic ultrasounds are used for evaluation and to exclude the possibility of malignant carcinoma.

Esophageal lipomatosis, as a distinct entity, was first described in 2000 in the Journal of European Radiology, where its occurrence was associated with steroid use [7]. Esophageal lipomatosis was described again in a 2002 case series, and its typical CT features were given the term the "double ring" esophageal sign [8]. The authors reported an incidence of only 7 among 1,555 (0.45\%) patients. The CT chest studies were performed primarily in men $(86 \%)$ with a mean age of 67 years. All the men had a clinical history of COPD, but only 4 were on chronic steroid therapy. Although our patient had a history of COPD, he was neither on steroid therapy nor was he officially diagnosed with Cushing's syndrome. Of note, one of the patients from the 2002 case series reported dysphagia that had been attributed to previous cerebrovascular accidents. The remaining patients were asymptomatic, like our patient. 
The typical appearance of fat on a CT scan is a well-circumscribed area with homogenous hypodensity with a thickness ranging from -70 to $-130 \mathrm{HU}$ [9]. In terms of location, esophageal lipomatosis commonly involves the proximal esophagus, as was the case in our patient. This tendency for the tumor to occur here might be due to striated musculature in the proximal esophagus. The appearance of esophageal lipomatosis on a CT scan consists of a low attenuation band, similar in opacity to subcutaneous fat and measuring negative Hounsfield units, located between 2 higher-density rings [9]. Although this may cause luminal narrowing, the clinical significance of this rare finding is not established.

With the increasing use of CT scan studies, it is important to recognize the benign nature of esophageal lipomatosis and to distinguish it from other esophageal lesions to avoid unnecessary invasive interventions. This has not yet been described in the GI literature. While endoscopy and endoscopic ultrasound scans would be the evaluations of choice for symptomatic or growing lesions, patients without symptoms and with the typical appearance of benign lesions on imaging may often only require clinical observation.

In conclusion, esophageal lipomatosis has only been described in a few case reports in the radiological literature and, to our knowledge has not been reported in the GI literature at all. Here, we describe an incidental finding of esophageal lipomatosis on CT imaging of the chest. The findings report esophageal lipomatosis as a benign entity that does not cause symptoms and usually does not warrant invasive diagnostic or therapeutic interventions, which justified the decision to clinically monitor the patient.

\section{Acknowledgments}

We thank all our nursing staff who took great care of our patient.

\section{Statement of Ethics}

Written informed consent was obtained from the patient for publication of this case report including patient's imaging studies. Ethical approval from the Institutional Review Board, VA Medical Center, Syracuse, NY, USA, was not required in accordance with local guidelines.

\section{Conflict of Interest Statement}

The authors have no conflicts of interest to declare.

\section{Funding Sources}

This study received no funding.

\section{Author Contributions}

Venkata Vinod Kumar Matli prepared preliminary draft and required data including imaging studies. Deepthi Devagudi revised, and Brian Cooney guided us in getting high-resolution images and explanation of the imaging findings. Uma Murthy revised and reviewed and then approved the article for submission.

\section{Karger'}




\section{Data Availability Statement}

All data generated or analyzed during this study are included in this article and its online suppl. Material Files (for all online suppl. material, see www.karger.com/ doi/10.1159/000519197). Further enquiries can be directed to the corresponding author.

\section{References}

1 Yoshimoto Y, Yoshida T, Fujikawa T, Shirai Y, Yamamoto T. Novel surgical approach without bowel resection for multiple gastrointestinal lipomatosis: a case report. Int J Surg Case Rep. 2019;59:54-7.

2 Bilgic Y, Altinsoy HB, Yildirim N, Alatas O, Kanat BH, Sahin A. Familial abdominal and intestinal lipomatosis presenting with upper GI bleeding. Case Rep Gastrointest Med. 2015 Jun2015;2015:1.

3 Gwak SY, Lee MK, Lee YK. A case of a bleeding duodenal lipoma successfully controlled by endoscopic resection. Clin Endosc. 2020 Mar;53(2):236.

4 Gao PJ, Chen L, Wang FS, Zhu JY. Ileo-colonic intussusception secondary to small-bowel lipomatosis: a case report. World J Gastroenterol. 2014 Feb;20(8):2117.

5 Sandhu PS, Bansiwal RK, Attri AK, Mittal R. Diffuse colonic lipomatosis, presenting as perforation peritonitis and mimicking carcinoma colon. Indian J Surg. 2011 Apr;73(2):155-7.

6 Ha C, Regan J, Cetindag IB, Ali A, Mellinger JD. Benign esophageal tumors. Surg Clin North Am. 2015 Jun;95(3): 491-514.

7 Bogaert J, Rosseel F, Verhaegen S, Verschakelen J. Esophageal lipomatosis: another consequence of the use of steroids. Eur Radiol. 2000 Aug;10(9):1390-4.

8 Marom EM, Goodman PC. Double-ring esophageal sign: pathognomonic for esophageal lipomatosis. J Comput Assist Tomogr. 2002 Jul;26(4):584-6.

9 Boiselle PM. Diffuse mediastinal abnormalities. In: McLoud TC, Boiselle PM, editors. Thoracic radiology: the requisites. Philadelphia: Mosby Elsevier; 2010. p. 369-78. 